\title{
Multiple Jumps and Vacancy Diffusion in a Face-Centered Cubic Metal
}

\author{
G. De Lorenzi $(*)(* * *)$ and F. ErColessi $(* *)(* * *)$ \\ (*) Centro di Fisica del Consiglio Nazionale delle Ricerche, I-38050 Povo, \\ Trento, Italy \\ (**) International School for Advanced Studies, I-34014 Trieste, Italy \\ (***) Materials Research Laboratory, University of Illinois at Urbana-Champaign, \\ Urbana, IL 61801, USA
}

PACS. 66.30F Self diffusion in metals, semimetals, and alloys.

PACS. 61.70B Interstitials and vacancies.

cond-mat/9208008 - To appear on Europhysics Letters. 
Abstract. The diffusion of monovacancies in gold has been studied by computer simulation. Multiple jumps have been found to play a central role in the atomic dynamics at high temperature, and have been shown to be responsible for an upward curvature in the Arrhenius plot of the diffusion coefficient. Appropriate saddle points on the potential energy surface have been found, supporting the interpretation of vacancy multiple jumps as distinct migration mechanisms. 
Several face-centered cubic metals, such as $\mathrm{Ag}, \mathrm{Cu}, \mathrm{Au}, \mathrm{Ni}$, and Pt, exhibit an upward curvature in the Arrhenius plot of the atomic self-diffusion coefficient close to the melting point [1]. To explain this effect, three mechanisms have been proposed [2]: (a) different types of defects (such as monovacancies and divacancies) contributing to the diffusion process with different activation energies [3]; (b) an intrinsic temperature dependence of enthalpy and entropy changes associated with the formation and migration of monovacancies [4]; (c) contributions from double jumps of monovacancies [5]. In this Letter we report molecular dynamics simulations in gold aimed at characterizing qualitatively and quantitatively the effect of correlated multiple jumps on the temperature dependence of the diffusion coefficient. Furthermore, from detailed studies of the potential energy surface, we provide a new microscopic interpretation of — at least — the double jump mechanism Ð.

It has already been known for some time that correlated jumps occur in the diffusion of vacancies in solids close to melting [5, 6, 7], but no statistically relevant measurements of this effect have been reported yet. Our results, based on the analysis of a large number of events, show that multiple jumps are present and very effective in bending the Arrhenius plot near the melting point. An interpretation of their microscopic nature has also been needed,

\footnotetext{
${ }^{1}$ It should be pointed out that our simulations do not address mechanisms (a) and (b) above, which therefore remain as further possible factors contributing to the curvature as suggested by some experiments.
} 
in order to integrate them into the framework of those recent developments in the theory of diffusion that explicitly take into account dynamical effects [8].

Empirical or semi-empirical many-body Hamiltonians developed in the last years have proved to be much more realistic for metals than pair potentials used in the past, in particular to describe surfaces or defects. The system we have used in our simulation consists of 255 particles with periodic boundary conditions, interacting via the "glue" many-body potential [9], which is well suited for our study since it reproduces rather well the melting temperature and the thermal expansion of $\mathrm{Au}$, as well as the $T=0$ energetics of a monovacancy. We have analyzed all the vacancy jumps in runs performed at twelve different temperatures, between $1000 \mathrm{~K}$ and $1550 \mathrm{~K}$, at steps of $50 \mathrm{~K}$. All the runs are done in the microcanonical ensemble, where temperature is defined by time averaging the total kinetic energy. At each temperature, the lattice spacing is obtained by an independent zero-pressure simulation of a perfect crystal. Since melting occurs near $1350 \mathrm{~K}$ in this model [9], runs at $1400 \mathrm{~K}, 1450 \mathrm{~K}, 1500 \mathrm{~K}$ and $1550 \mathrm{~K}$ refer to an overheated crystal which, in the absence of free surfaces, remains stable within the simulation time scale (mechanical instability was reached around 1600K). We find these runs to be very useful to observe the trends of the diffusion mechanisms at high temperatures, even if they cannot be directly compared with experimental results. With the exception of the runs at $1000 \mathrm{~K}, 1050 \mathrm{~K}$ and $1100 \mathrm{~K}$, where 
the jump rate is particularly low, the runs are made long enough to contain $\sim 800$ jumps in order to obtain sufficient statistics of rare correlated jumps. This requires a run length of $57 \mathrm{~ns}\left(8 \times 10^{6} \mathrm{MD}\right.$ steps $)$ at $T=1150 \mathrm{~K}$, and progressively shorter runs at higher temperatures.

From the Arrhenius plot shown in fig. 1 (filled circles), it can already be seen that migration of monovacancies alone - the only type of defect present in our simulation-produce a measurable curvature. The curve bends just below $T_{m}$ and continues with a higher slope into the superheated region. The quantity plotted is the atomic migration contribution to the diffusion coefficient, defined as

$$
D=\frac{\left\langle u^{2}(t)\right\rangle-\left\langle u_{\mathrm{bulk}}^{2}\right\rangle}{6 t}
$$

where $t$ is the run length, $u^{2}(t)=|\mathbf{r}(t)-\mathbf{r}(0)|^{2}, u_{\text {bulk }}^{2}$ is the asymptotic value of the same quantity for a perfect bulk (not diffusing), independently calculated and subtracted in order to retain only the diffusive part, and the angular brackets indicate average over all the atoms. The atomic diffusion coefficient, as usually defined, is $D$ multiplied by the ratio of the concentration of vacancies in the system at the given temperature to the concentration in our model system (fixed to be 1:256 at all temperatures).

To determine the presence and role of vacancy multiple jumps, we have analyzed all the jump events with respect to both their relative times of occurrence and of the atomic displacements involved. In a vacancy multiple jump two or more atoms move simultaneously. Each atom moves by only one 
lattice spacing, $d$, but the vacancy, as a result of this concerted motion, is displaced by one or more lattice spacings, depending on the number of atoms involved and their relative directions. In a double jump, for example, if the two atoms move in the same direction the vacancy is displaced by $2 d$, but if the two atoms move at an angle of $120^{\circ}$ the vacancy will be displaced by only $d$.

We have first analyzed the jump events in terms of their relative times of occurrence. At each time step, the occupation number of each Wigner-Seitz cell in the fcc lattice is computed. Every change in the occupation numbers ("event") is recorded, along with the time step at which it occurs. A large fraction of the jumps has been found to be followed almost immediately [i.e., within a Debye period, corresponding to about 40 MD steps (0.29 ps)] by a return jump into the original position. It is very likely that these event pairs are not real jumps, but rather occasional excursions of a neighbouring atom into the cell containing the vacancy. Such jumps are considered as miscounts and eliminated from the analysis. If we consider all the jumps followed by a return jump within a time $\tau$ and calculate the average delay $\delta$, we observe that when $\tau$ becomes larger than about 50 steps (0.36 ps), $\delta$ tends to stabilize around 25 steps, nearly independently of temperature. We have therefore taken $\tau_{R}=25$ steps $(0.18 \mathrm{ps})$ as the average delay of immediate returns, and removed all the jumps followed by a return within $3 \tau_{R}=75$ steps. Assuming an exponential distribution, this should eliminate 
about $95 \%$ of these miscounts. After this filtering, the distribution of delays between successive jumps at $T=1450 \mathrm{~K}$ is shown in fig. 2. For large times the semi-logarithmic plot is linear, as expected for uncorrelated jumps. For delays of less than 200 steps (1.43 ps), however, strong deviations from linearity indicate the presence of correlated jumps. The same feature, even if not so pronounced, is found also at the lower temperatures, down to $1250 \mathrm{~K}$. Qualitatively similar results have been found in a simulation study of vacancies in $\mathrm{Al}$ [5]. Thanks to the much larger statistics of events, we can here provide statistically valid information about their contribution to the Arrhenius plot, and we also provide an interpretation of their microscopic mechanism.

We have classified all jumps into single, double, triple, etc., by grouping together those events separated in time by less than $\tau_{M}=200$ steps. The temperature dependence of the frequencies for the different groups is reported in fig. 3, referring to the range from $1150 \mathrm{~K}$ to $1450 \mathrm{~K}$ [computational limitations prevented us to obtain sufficient statistics of multiple jumps for $T<1150 \mathrm{~K}$, while at $1500 \mathrm{~K}$ and $1550 \mathrm{~K}$ (far into the superheated region) correlated jumps become so complex to make this analysis impractical to do]. The apparent activation energies are not simple multiples of the energy for single jumps, as would be expected if they were extracted from a distribution of uncorrelated jumps. The contribution $D_{S}$ of single jumps to the diffusion coefficient, represented by the open circles in the Arrhenius plot of 
fig. 1, even exhibits a small downward curvature at the highest temperatures (superheated region), in contrast with the upward curvature of the total $D$. From this second step of our analysis, it is clear that the upward curvature observed in our simulation is due to correlated jumps.

We have proceeded in analyzing these correlated events in terms of the angles between the directions of successive vacancy jumps. Reasonable statistics was available only for double jumps. In the fcc lattice there are five possible angles between the directions of two vectors joining three consecutive lattice sites: $0^{\circ}$ (collinear), $60^{\circ}, 90^{\circ}, 120^{\circ}$ and $180^{\circ}$ (return jumps). Given a vacancy in a specific lattice site, there are $m$ equivalent ways it can perform a given double jump, where $m=12,48,24,48$, and 12 for $0^{\circ}, 60^{\circ}, 90^{\circ}, 120^{\circ}$ and $180^{\circ}$ double jumps, respectively. For a series of random jumps, the frequency, $\Gamma$, divided by the multiplicity, $m$, should then be uniformly distributed among the five angles. This is what we find, within the statistical uncertainty, for pairs of jumps with delays $\tau>\tau_{M}$. But for $\tau<\tau_{M}$ the distribution is rather

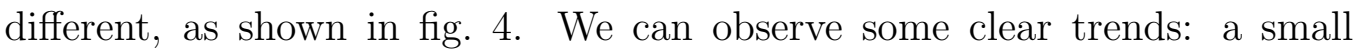
increase above the random ratio for $0^{\circ}$, a definite increase for $60^{\circ}$, a small decrease for $90^{\circ}$ and a definite decrease for $120^{\circ}$ double jumps. $180^{\circ}$ double jumps also increase, but behave quite erratically with temperature. These last events correspond to return jumps of the same atom and do not contribute to the diffusion. In agreement with the previously given definition, in the following we will call double jumps only those events involving two atoms. 
The apparent activation energies for the separate groups are: 1.9, 1.6, 1.9 and $1.6 \pm 0.2 \mathrm{eV}$ for $0^{\circ}, 60^{\circ}, 90^{\circ}$ and $120^{\circ}$ double jumps, respectively.

The question now arises if these correlated jumps correspond to dynamical effects or to different jump mechanisms [7]. The first situation would imply the fast successive crossing of two single jump barriers in series, the latter the crossing of a different, higher barrier, specific for each double jump. To shed some light on this point, we have thoroughly investigated the potential energy surface in $3 N$-dimensional space [10], around possible configurations for double jump saddle-points. We proceeded by trial and error, first looking for watersheds between equilibrium configurations with minimization techniques, and then looking for extrema in the intermediate regions, always letting all the $3 N$ atomic coordinates relax in this process. The calculation has been done at the equilibrium density for $T=1350 \mathrm{~K}$. Relatively low energy paths for double jumps can in fact be found. The corresponding saddles are not as simple as the traditional saddle point for single jumps (fig. 5), which is quadratic and has only one unstable normal mode, but are instead combinations of minima, saddle points and crossroads (saddle points with two unstable normal modes), as shown, e.g. for the case of $0^{\circ}$ double jumps in fig. 6. However, the difference in energy between these local structures is never larger than $0.02 \mathrm{eV}$. The energies of the lowest points in the barriers for the various double jumps with respect to equilibrium are: 1.93, 1.92, 2.29 and $2.66 \mathrm{eV}$ for $0^{\circ}, 60^{\circ}, 90^{\circ}$ and $120^{\circ}$ double jumps, respectively. These val- 
ues are to be compared with the apparent activation energies evaluated from MD and reported above. Taking into account the errors in the MD estimates, and the fact that such a comparison is valid only at low $T$, the energies for $0^{\circ}, 60^{\circ}$ and $90^{\circ}$ jumps can be considered rather close. This agreement is an indication that double jumps constitute distinct jump mechanisms, corresponding to the passage over characteristic energy barriers. In fact, we have also verified that the distance between the two jumping atoms remains close to the first-neighbour distance throughout the jump, in most $0^{\circ}, 60^{\circ}$ and $90^{\circ}$ jumps. The MD estimate of the activation energy for $120^{\circ}$ jumps is, on the other hand, sensibly lower than the height of the barrier found for this mechanism. However, the frequency of $120^{\circ}$ jumps is much smaller than the other types of double jumps, and these events cannot really be distinguished from those that would be expected for a distribution of pairs of successive uncorrelated single jumps.

Finally we note that the anomalous number of return jumps could be due to the complicated structure of the saddles, where trajectories can be momentarily trapped and then turn back. As shown by fig. 6, successful completion of a double jump also requires the unstable normal mode to change near the saddle point. Moreover, we have found a second family of saddle points for single jumps on the sides of the traditional one (fig. 5). In these, the jumping atom is still placed halfway between the two half-vacancies, but, instead of lying in the (100) plane, it sits either below or above it by about 
$30 \%$ of the nearest-neighbour distance. These lateral saddles have the same complicated structure as the saddles for double jumps (fig. 6), with a saddle point energy of $1.18 \mathrm{eV}$, and a local minimum of $1.15 \mathrm{eV}$, lower than the energy of the traditional saddle point for single jumps, $1.34 \mathrm{eV}$.

In summary, the migration of monovacancies in gold, as simulated by molecular dynamics, exhibits an Arrhenius plot with a pronounced upward curvature at high temperature. This curvature is due to multiple jumps, in which two or more atoms jump simultaneously. An analysis of the potential energy surface reveals the presence of distinct saddle points appropriate for double jumps, and supports the interpretation of correlated jumps as distinct migration mechanisms.$$
* * *
$$

Useful comments by Gert Ehrlich, Giulia Galli, Gianni Jacucci, Enrico Smargiassi and by a referee are gratefully acknowledged. This work has been performed partly under the Progetto Finalizzato CNR "Sistemi informatici e calcolo parallelo" and under the U.S. Department of Energy Grant No. DEFGO2-91ER45439.
\end{abstract}




\section{References}

[1] Herzig C., Eckseler H., Bussmann W., and Cardis D., J. Nucl. Mat. 69/70 (1978) 61; Sahu R. P., Jain K. C., and Siegel R. W., J. Nucl. Mat. 69/70 (1978) 264.

[2] Mehrer H., J. Nucl. Mat. 69/70 (1978) 38; Peterson N. L., J. Nucl. Mat. 69/70 (1978) 3; Neumann G. and Tolle V., Phil. Mag. A 54 (1986) 619; see also Materials Science Forum 15/18 (1987).

[3] Peterson N. L., Comm. Solid State Phys. 8 (1978) 107.

[4] Gilder H. M. and Lazarus D., Phys. Rev. B 11 (1975) 4916.

[5] Da Fano A. and Jacucci G., Phys. Rev. Lett. 39 (1977) 950.

[6] Bennett C. H. and Alder B. J., J. Phys. Chem. Solids 32 (1971) 2111; Jacucci G., in Nontraditional Methods in Diffusion, edited by G. E. Murch, H. K. Birnbaum and J. R. Cost, (The Metallurgical Society of AIME, New York, 1984), p. 259.

[7] See e.g. the appendix in: Flynn C. P. and JACucci G., Phys. Rev. B 10 (1982) 6225.

[8] Toller M., Jacucci G., De Lorenzi G., and Flynn C. P., Phys. Rev. B 32 (1985) 2082. 
[9] Ercolessi F., Tosatti E., and Parrinello M., Phys. Rev. Lett. 57 (1986) 719; Philos. Mag. A 58 (1988) 213.

[10] See, e.g., JaCuCCI G., in Diffusion in Crystalline Solids, edited by G. E. Murch and A. S. Novick, (Academic Press, New York, 1984). 


\section{Figure Captions}

Figure 1. Atomic migration contribution to the tracer diffusion coefficient $D$ via monovacancies. Filled circles indicate $D$ as measured directly from Eq. (1); diamonds indicate $D_{\text {all }}$ as evaluated from the jump frequency $\Gamma$, with all the jumps treated as if they were single, uncorrelated jumps; and open circles indicate the contribution $D_{S}$ from only single jumps. $d$ is the nearest-neighbour distance and $f_{S}=0.78146$ is the geometrical correlation factor for single jumps. Apparent activation energies are shown.

Figure 2. Time delays between successive jumps of the vacancy. The bin width is 200 steps.

Figure 3. Frequencies of single (S), double (D), triple (T) and quadruple (Q) jumps. Error bars correspond to a standard deviation. The lines, whose slopes are given as activation energies, are obtained by a weighted fit.

Figure 4. Distribution of angles between the jump directions of pairs of successive jumps. The jump frequency $\Gamma$ is divided by the geometrical multiplicity $m$ (see text).

Figure 5. Saddle on the $3 N$-dimensional potential energy surface for single jumps. The plot shows a section of the hypersurface along the directions of the unstable normal mode (the jumping mode), $\eta$, and the softest 
stable normal mode, $\zeta$. Lengths along $\eta$ and $\zeta$ are in $\AA$ and the energy is reported relatively to the energy of the equilibrium configuration. The atomic positions in the saddle point configuration and the atomic displacements corresponding to $\eta$, projected onto the (100) plane, are also shown. Note that $\eta$ and $\zeta$ are both $3 N$-dimensional vectors, but their components along the cartesian coordinates of the nonjumping atoms are so small that cannot be detected in the figure.

Figure 6. Saddle for $0^{\circ}$ double jumps. Conventions are as in fig. 5. The central configuration is a local minimum. $\zeta$ is the direction of the softest mode. Along the second axis, in order to clearly show the relevant structures of the energy surface, we have chosen two different halfdirections $\eta_{-}$and $\eta_{+}$for the negative and positive part. This is why there is a sharp edge where the two sections meet. The two sketches on the left show the atomic displacements in the crossroad positions. Direction $\eta_{-}$corresponds to the first half of the jump: the atom in front is slower than the one behind it, so that their separation decreases (as also seen by the overlap of the two jumping atoms). Direction $\eta_{+}$ corresponds to the second half of the jump: the atom in front is faster. Completion of a double jump therefore requires a change of the unstable normal mode to occur near the central position. 